\section{Wood - An Innovative Component of High-tech Products? 6th Wädenswil Day of Chemistry 2014}

Elsbeth Heinzelmann, science + technology journalist

Abstract: Wood is a multifunctional renewable material that could replace oil-based and other non-renewable resources in many applications. In the Swiss National Science Foundation project NFP 66, entitled 'Wood as a resource', researchers are attempting to find ways to use wood more efficiently in the future. The 6th Wädenswil Day of Chemistry, which was organised by the Institute of Chemistry and Biological Chemistry at Zurich University of Applied Sciences in Wädenswil, gave an overview of the innovative activities currently being undertaken in this field.

Keywords: Cellulose nanocrystals · Polyflavonoid tannins . Switchable membranes - Wood-decomposing fungi

\section{A Closer Look at Wood-decomposing Fungi}

Wood could be of great service to us as a component of chemical products and through wood-based materials such as fibres and lignin derivatives. However, although more than $90 \%$ of the world's biomass consists of wood, it is not being used for chemical syntheses because it is not readily biodegradable. In other words, wood is a poorly used resource. In order to change this, a group led by Professor Florian Seebeck in the Chemistry Department of the University of Basel is investigating secondary metabolites in fungi that support the lifestyle of Serpula lacrymans or Dichomitus squalens, for example, which cause wood to decay.

The focus is on lignocellulose, a compound of partially crystalline polysaccharides (cellulose and hemicellulose) and polyaromatic resin (lignin). This physically efficient and chemically inaccessible material is a successful component in the development of land plants that compete with each other as they grow towards the sunlight. It is derived from air, light and water, absorbs minimal amounts of often limiting elements such as nitrogen or phosphorus and has shown itself to be largely resistant to microbial decomposition.

"Around 300 million years ago, fungi started to integrate this widely available unused source of nutrients into a tight carbon cycle: enzymes that decompose lignin, such as lignin peroxidase and manganese peroxidase, directly mineralised lignin into $\mathrm{CO}_{2}$," explains Florian Seebeck, who is an active participant in the NFP 66 project. "By decomposing lignin, fungi - together with numerous soil bacteria - gain access to cellulose and hemicellulose as oxidisable organic material." Much of the 50 billion tonnes of lignocellulose that grows annually is thus recycled in the biosphere. "Interestingly enough, this globally important biochemical process arose relatively late in terms of natural history, and only in response to an accumulating unused resource," says the Professor of Molecular Bionics. "It is equally striking that little is known about the microbial decomposition of

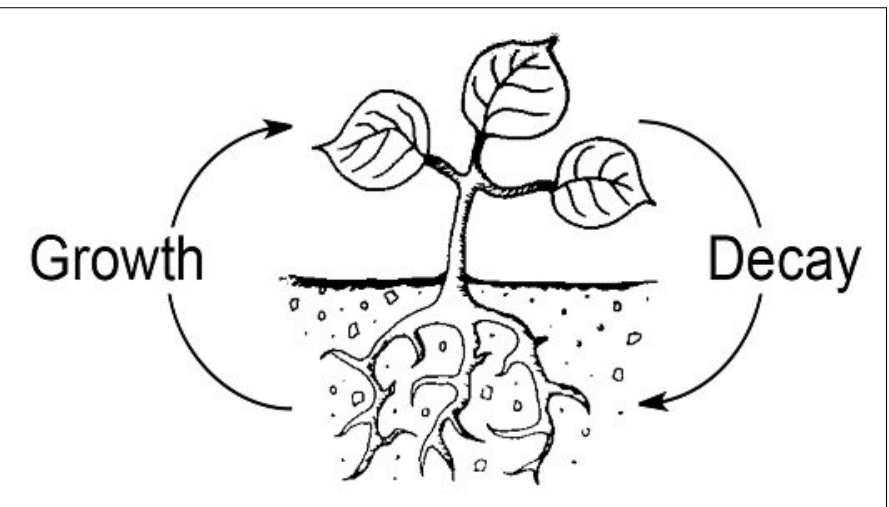

Biological cycle of lignocellulose. Lignocellulose is derived from water, air and sunlight by a process of photosynthesis. Its remineralisation into $\mathrm{CO}_{2}$ and water is a diverse process in which saprophytic fungi and bacteria play a key role that is insufficiently understood. The mechanisms involved could point the way to new potential uses for biomass. Source: Prof. Florian Seebeck, Basel University

biomass, even though this process could show how biomass can be used to produce fine chemicals, building materials and fuels."

In order to investigate these processes, the researchers have to overcome a number of challenges: "On the one hand, wood decomposition is the result of cooperation between various organisms, enzymes and small molecules. The traditional focus on an active principle, species or catalytic activity fails to throw light on this complex topic. On the other hand, in order to understand how wood decays we need to integrate the chemical-molecular as well as the nano and microscopic aspects. And finally, saprophytic fungi, the actual protagonists of wood decomposition, are comparatively complex organisms that we have only just started to understand better in the last few years thanks to intensive genome sequencing programmes." (F. P. Seebeck, J. Am. Chem. Soc. 2010, 132, 6632; F. P. Seebeck, Chimia 2013, 67, 333.)

\section{Cellulose Nanocrystals from Banana Plants}

It was the French chemist Anselme Payen who discovered cellulose in 1838 and isolated it from plants. This polymer is the principal component of plant cell walls, the most common organic compound and also the most common polysaccharide. Cellulose molecules form higher structures which produce tear-resistant fibres that can be used in paper manufacturing, for example. "Where cellulose macromolecules are aligned on a single axis, highly crystalline areas form, interspersed with amorphous material," explains Prof. Christoph Weder of the Adolphe Merkle Institute (AMI) at the University of Fribourg. "If you hydrolise natural cellulose material controlled with mineral acids such as sulphuric, hydrochloric or phosphoric acid, the amorphic parts dissolve and you get cellulose nanocrystals (CNCs) with a well-defined outline." The Professor of Polymer Chemistry and Materials and Director of the AMI wants to isolate these CNCs because they can be used as cheap, bio-based and highly rigid fillers for polymer nanocomposites. CNCs are highly 
crystalline nanoparticles with a rod-like appearance, a diameter of 5-20 nm and a length of between 100 and $3000 \mathrm{~nm}$. They have amazing mechanical properties: in addition to high rigidity and strength they also demonstrate a high aspect ratio. "CNCs are now an attractive component for a wide range of nano materials, such as mechanically adaptive materials, aerogels, optically or electrically active materials and numerous mechanically reinforced polymer nanocomposites," explains Christoph Weder. "CNCs can be extracted from almost all natural cellulose material, but characteristic properties such as the maximum aspect ratio, crystal structure and crystallinity vary according to origin."

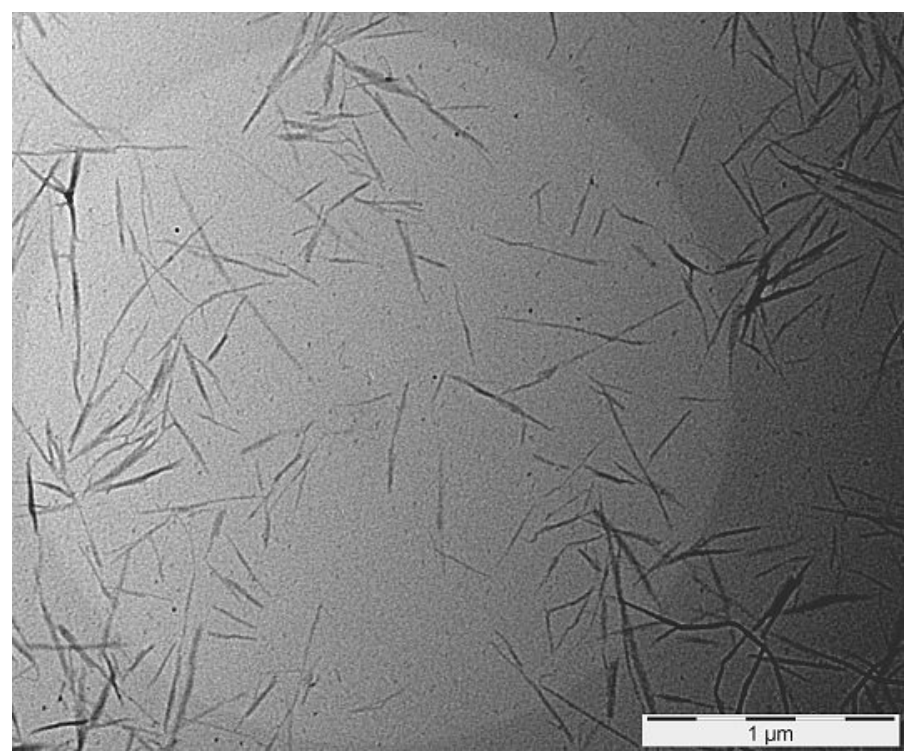

Transmission electron microscopy of cellulose nanocrystals, extracted from banana stems. Hydrolysis period: 90 minutes. Image AMS/UNIFR

In projects funded by the Swiss National Science Foundation as part of the National Research Programmes "Opportunities and risks of nanomaterials" (NFP 64) and "Wood as a resource" (NFP 66), the scientist and his team investigated the isolation of CNCs from natural materials such as banana plants and developed simple processing methods to integrate them into conventional polymers. The dried stems of Musa sapientum Linn were first cleaned by Soxhlet extraction, then treated with alkali and bleached, and finally hydrolised into CNCs using sulphuric acid. The hydrolysis period was systematically varied with the aim of maximising the length and aspect ratio of the CNCs. The surface charge density of the isolated CNCs was $168 \mathrm{mmol} \mathrm{kg}{ }^{-1}$, the predominant crystal structure was that of cellulose 1 and the crystallinity was $74 \%$. In order to verify the reinforcement properties of these CNCs, nanocomposites of an ethylene oxide-epichlorohydrin copolymer were prepared and their mechanical properties investigated using dynamic mechanical analysis (DMA). Doctoral student Silvana Müller is pleased with the progress so far: "A comparison with reference nanocomposites prepared with $\mathrm{CNCs}$ isolated from cotton shows that these new CNCs demonstrate greater - and exceptionally high - reinforcement stability and a significantly better aspect ratio."

\section{Bark from Softwoods Used in Natural Adhesives}

Tree bark as a by-product of the wood processing industry offers as yet untapped commercial potential. Polyflavonoid tannins are key components in the production of natural adhesives. Researchers are therefore conducting a project to develop a process for obtaining tannins from the bark of native softwoods. Under the guidance of Dr. Frédéric Pichelin, Head of the Institute of Wood Science and Technology in Biel, the Bern University of Applied Sciences is cooperating with the Zurich University of Applied Sciences, Wädenswil, as well as with Kronospan Switzerland AG (a manufacturer and refiner of wood materials), Perlen Papier AG (a manufacturer of publication papers) and the Centre for Wood Science of the University of Hamburg.

The first task is to develop an extraction process for tannins that can be implemented in the material flow of industrial processes - such as in sawmills. The focus is on the selective preparation of bark compounds in order to develop a production method for formaldehyde-free adhesives. The tree species being investigated are the spruce (Picea abies), the pine (Pinus sylvestris), the European larch (Larix decidua), the European silver fir (Abies alba) and the Douglas fir (Pseudotsuga menziesii). In order to characterise the chemical properties of the phenolic compounds with the potential to make wood glue, the scientists extracted ground bark by means of aqueous solutions. They then combined an HPLC UV analysis with thiolysis. "We were thus able to identify the components and the degree of polymerisation of the condensed tannins extracted."

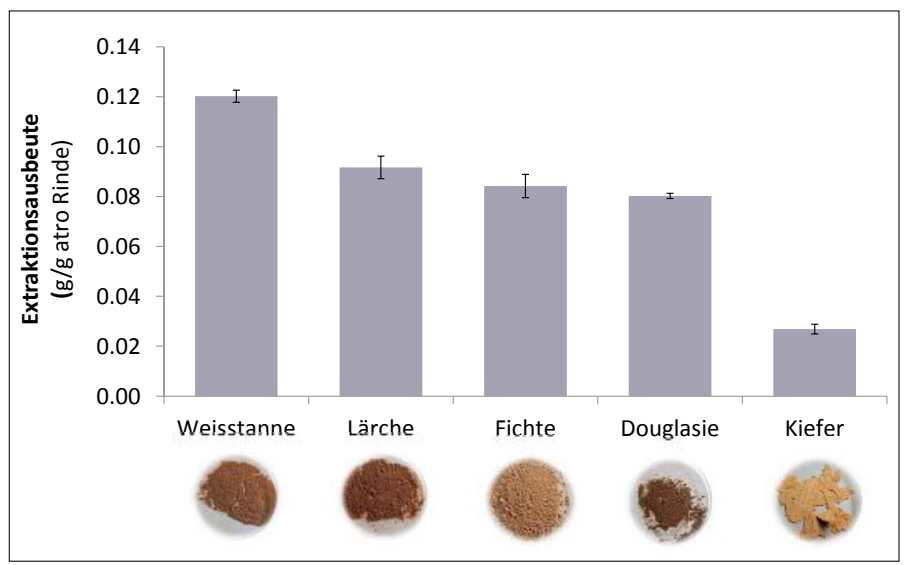

Prof. Frédéric Pichelin's project focuses on the development of a customised process for extracting bark tannins from native softwoods. The aim is to produce tannins as adhesives in wood-based materials production. Image BFH-TI

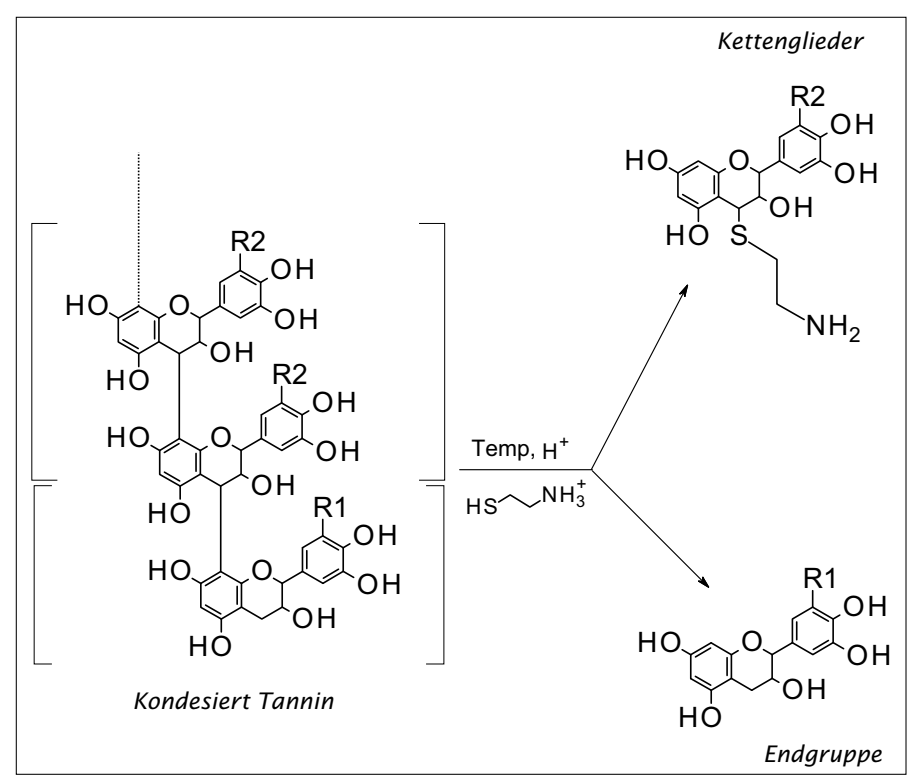

Thiolysis condensed tannins in the presence of cysteamine hydrochloride. Base polymer and resulting separated end units and extension units. Image BFH-TI

The MALDI-TOF MS process, which is used for the structural characterisation of polymers, was used to characterise the bark tannins. "As our investigations show, the properties of tannins vary according to composition, structure and degree 
of polymerisation," explains Frédéric Pichelin. "That's why we are now examining the influencing extraction parameters such as solvents, additives, temperature and extraction time." The extracted tannins are being evaluated on this basis and the extraction process optimised. Combining different tannin extracts allows an adhesive system to be tailored to the specific requirements of the wood-based material production.

\section{Using Wood as a Material Adds Value}

Wood is one of the oldest building materials in the world, but it burns, absorbs water, shrinks and often has a short life span. In order to use it more efficiently, processes have hitherto aimed at increasing its dimensional stability and protecting wood from natural degradation. "Although this improves some properties of wood, most processes reduce the functional access to the cell wall polymers, which makes it difficult to use wood in new applications," explains Ingo Burgert, Professor of Wood-based Materials at ETH Zurich and head of a research group in the Department of Applied Wood Research at Empa. He seeks ways to create added value by combining wood and biomaterial research with nano and polymer technology. He and his colleagues use in situ polymerisation to transform the wood cell wall into a refined anisotrophic composite structure with excellent mechanical properties, based on oriented cellulose fibrils embedded in a matrix made of hemicellulose and lignin.

In one of the modification approaches, wood is functionalised using an azo initiator which penetrates the wood cell wall and reacts with the hydroxyl groups of the cell wall components. The azo initiator embedded in the wood enables the radical polymerisation of impregnated monomers (vinyl and acrylate). Polymer chains grow within the cell wall cavities and form a thin polymer layer which also covers the inner surface of the cell wall. "This approach allows novel functionalities to be implemented in the renewable and sustainable material that is wood," says Etienne Cabane, who developed this modification approach and is a member of the research group. The original wood structure is retained and used as a hierarchical multi-scale reference system for a modular two-stage polymerisation process. It may therefore be possible to control the micropore filling of the wood through the composition and thickness of the polymer layers on the inner surface of the cell wall. These can be used to develop wood-based switchable membranes which exploit the natural porosity of the wood for separation purposes.

\section{The ICBC Builds Bridges}

By organising the 6th Wädenswil Day of Chemistry, the Institute of Chemistry and Biological Chemistry (ICBC) is demonstrating its commitment to a lively exchange of views on the theme of wood. "We are particularly pleased to have an opportunity to strengthen the bridge between pure and applied science," says Professor Christian Hinderling, the Institute's director. "ICBC believes that wood, as a renewable resource, is a highly topical subject with regard to new materials and also as a source of raw and constituent materials for 'green chemistry'."

\section{Further information:}

Prof. Dr. Christian Hinderling

Life Sciences and Facility Management ZHAW

CH-8820 Wädenswil

E-mail: christian.hinderling@zhaw.ch

www.lsfm.zhaw.ch/de/science/institute-zentren/icbc.html



\section{Wood cell wall grafted with polystyrene}

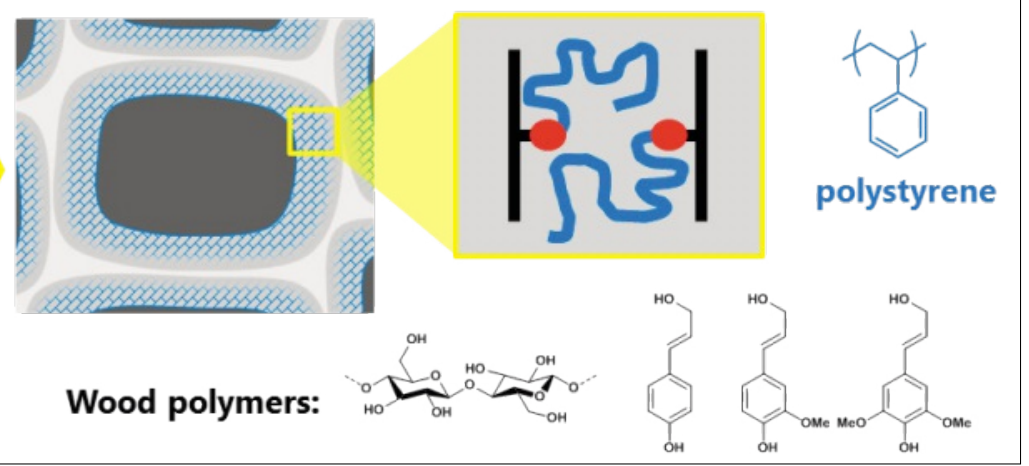

Wood cell wall grafted with polystyrene. Image ETHZ 